\title{
Multifocal central nervous system hemangioblastoma: a case report and review of the literature
}

\author{
L.Z. Chu', ${ }^{1,3}$ Z.Z. Guan ${ }^{1,2}$, J. Liu ${ }^{3}$, H. Yang ${ }^{3}$, X.L. Qi ${ }^{2}$, M.G. Dong ${ }^{3}$, \\ Y.M. Chen ${ }^{3}$, Y.N. Xiang ${ }^{1}$ and Y. $\mathbf{L i}^{2}$ \\ ${ }^{1}$ Department of Pathology, Guiyang Medical University, Guiyang, China \\ ${ }^{2}$ Department of Molecular Biology, Guiyang Medical University, \\ Guiyang, China \\ ${ }^{3}$ Department of Neurosurgery, \\ The Affiliated Hospital of Guiyang Medical College, Guiyang, China
}

Corresponding author: Z.Z. Guan

E-mail: zhizhongguan@yahoo.com

Genet. Mol. Res. 13 (3): 7904-7911 (2014)

Received June 18, 2013

Accepted January 14, 2014

Published September 29, 2014

DOI http://dx.doi.org/10.4238/2014.September.29.3

\begin{abstract}
An effective therapy for multifocal central nervous system hemangioblastoma (CNS HB) is needed. Here, we report a case of multifocal CNS HB. A 43-year-old man was diagnosed with CNS HB by enhanced computed tomography and magnetic resonance imaging. Six solid tumors and one cystic nodule were detected in his cerebellum. The patient underwent three surgeries followed by knife radiosurgery and had regular visits after the operation. In addition, histological observation with hematoxylin and eosin staining and immunohistochemistry for $\alpha$-inhibin, $\mathrm{Ki} 67$, and vascular endothelial growth factor further provided evidence of cerebral HB. The symptoms of the patient were prominently improved after each operation, suggesting that multiple surgeries and radiation therapy are needed to prevent the proliferation and relapse of multifocal CNS HB. In addition, long-term, regular hospital visits were useful. Furthermore, genetic
\end{abstract}


diagnosis and gene-targeted therapy might be a promising strategy against familial CNS HB in the future.

Key words: Multifocal central nervous system hemangioblastoma; Radiosurgery; Post-operation visit

\section{INTRODUCTION}

The histological causes and mechanisms leading to morbidity in patients with central nervous system hemangioblastoma (CNS HB) remain unclear. The malignancy often arises as a single focus in the cerebellum, whereas multifocal CNS HB is rare and extremely difficult to cure. Multifocal CNS HB is a family hereditary disease; it may arise in conjunction with von Hippel-Lindau (VHL) syndrome, in which neoplasms develop in several organ systems (Ma et al., 1998). Clinical treatment for multifocal CNS HB faces two obstacles: 1) multiple pathological changes occur simultaneously in the cranium and 2) the ectopic recurrence of tumors is difficult to control. In addition, as a component of VHL syndrome, multifocal CNS HB is accompanied by tumor generation in other tissues and organs throughout the patient's body (Woodward et al., 2007).

From March 1997 to November 2011, 8 patients (5 males and 3 females) from four familial generations were admitted to the affiliated hospital of Guiyang Medical University and were diagnosed with CNS HB. Of these, 2 patients had multifocal CNS HB, including a male patient whose case is extensively discussed in this report. Specifically, the patient had typical symptoms of multifocal CNS HB with a convoluted condition.

\section{CLINICAL PRESENTATION}

\section{Diagnosis and gene screening}

When the patient first visited our hospital, his chief complaints were headache and vertigo. At his second and third visits, he mainly complained of headache and an unsteady gait, which was attributed to increasing intracranial pressure. The diagnosis was confirmed later by enhanced computed tomography (CT) and magnetic resonance imaging (MRI). Imaging analyses identified seven tumors, including six solid tumors and one cystic nodule. The solid tumors ranged in size from $2.0 \times 2.5 \times 2.5$ to $4.5 \times 5.0 \times 5.0 \mathrm{~cm}$, and they were pink in color and soft or moderately hard in texture. Obvious thickening in the feeding artery and venous return was observed. The cystic nodule displayed a peanut kernel-like shape. In addition, three of the tumors were rooted in the cerebellar hemisphere, three tumors were located in the vermis and annular pool, and the last tumor was located on the dorsal face of the medulla oblongata. The multifocal characterization of this patient was familial and indicative of VHL syndrome. Considering that his family had a history of VHL disease, the patient was unequivocally diagnosed with VHL syndrome.

It has been reported that sporadic cerebral hemangioblastoma is caused by mutations in a certain chimera of the VHL gene (Jarrell et al., 2006). Analyzing gene mutations in the relatives of patients with VHL syndrome who do not have a significant history or symptoms of the disease would permit the early identification of potential pathogenic gene carriers. Therefore, it is necessary to perform gene screening for all family members of patients with VHL syndrome (Rasmussen et al., 2006). With the expectation of effectively administering early treatment and improving the progno- 
sis, individuals who present positive results on the test should be thoroughly examined in the clinic and observed both in and out of the hospital. As no mutation was identified in the chromatin test for our patient, we only selected a few methods of detection. Meanwhile, two asymptomatic individuals in the family of the patient were examined by imaging and VHL gene detection tests.

VHL syndrome is characterized by accumulated morbidities in multiple organs, including cerebral hemangioblastoma. New tumors tend to arise anywhere in the body throughout the patient's lifetime (Miyagami and Katayama, 2004). It is important to perform examinations, including abdominal ultrasound examination, CT, MRI, ophthalmoscopy, and blood cell counts, to assess the patient's condition as a whole. No pancreatic cyst or renal tumor was found in the case of our patient. The blood cell count revealed a red cell count of $4.34 \times 10^{12} / \mathrm{L}$ and a hemoglobin concentration of $156 \mathrm{~g} / \mathrm{L}$. Chromosomal examination of 320-400 bands did not reveal any abnormal changes in the patient's peripheral blood. However, the possibility of microdeletion and abnormal insert could not be excluded.

\section{Radiosurgery and regular post-operation visits}

Briefly, radiosurgery supplemented by Gamma knife radiosurgery is our core therapeutic method for treating these tumors. Considering the high incidence of reoccurrence, regular patient visits after the primary operation were employed to ensure that he could receive timely treatment in case of reoccurrence.

The patient underwent three transcranial microsurgeries. In the first operation in December 2005, one solid tumor and one cystic nodule were excised, and shunting of the lateral ventricle and spinal subarachnoid space was accomplished. The nodule, especially those hide in the cyst cavity or cyst wall, were located by multi-axis positioning and enhanced MRI prior to surgery. During the course of the operations, Omega Navigation System support was sought. The shapes of multifocal cyst nodules were exactly localized, as the nodules appeared to have a multi-azimuth appearance (Figure 1). The nodules invaded cyst cavities, penetrated the cyst wall, or remained outside the cyst wall. An uncut nodule may retain the ability to produce cyst fluid and ultimately induce tumor regeneration (Miyagami and Katayama, 2004; Jagannathan et al., 2008). Therefore, we attempted to remove the cyst wall and the nodule. In the second surgery in September 2006, three tumors were excised (Figure 2). Residual lesions were treated by Gamma knife radiosurgery 9 months after the surgery. In addition, before the third craniotomy surgery, brain digital subtraction angiograph (DSA) testing was performed. In this operation, the last solid tumor was removed (Figure 3).

Solid tumors were removed in entirety according to the following sequence of events: the tumor-feeding vasculature outside the tumor capsule was isolated; resecting the feeding artery by electric coagulation; manipulations were kept close on the tissue body to avoid removing the draining vein from the shallow to the deep end; we waited until the color of the tumor faded, the tension decreased, and the tumor reduced in size; and then the veins were last removed (Lee et al., 2003). For large solid tumors or those rooted into the brain stem, thorough extirpation cannot be achieved. The rich blood feeding system of such neoplasms may make operations more difficult. Puncturing or blocking resection should be avoided in cases of uncontrollable massive hemorrhage. However, no significant adhesion was observed between the solid tumors and vital structures of the brain such as the brain stem and the four ventricles. Thus, complete removal could be pursued. In cases of multiple nidus or deep 
roots, stereotactic radiosurgery Gamma knife treatment should be performed because the lesions are not sensitive to conventional radiotherapy.

After each operation, the levels of $\alpha$-inhibin, Ki67, and vascular endothelial growth factor (VEGF) in the excised tissues were measured by immunohistochemistry using the appropriate monoclonal antibodies, and the expression of these proteins was detected by the method of SP. The results revealed a 55\% (+++) increase in $\alpha$-inhibin expression, a $12.9 \%$ $(+++)$ elevation in Ki67 expression, and high VEGF expression (six points) (Figures 4 and 5). The upregulation of Ki67 and VEGF further proved the accuracy of the method for detecting cerebral hemangioblastoma. We concluded that the high expression of these two factors is predictive of tumor recurrence after surgery.

In March 2007, the third operation was performed. During the operation, the patient was exposed to radiation therapy with a central dose of $28.2 \mathrm{~Gy}$ and a peripheral dose of 11.6 Gy. The isodose was $55 \%$.

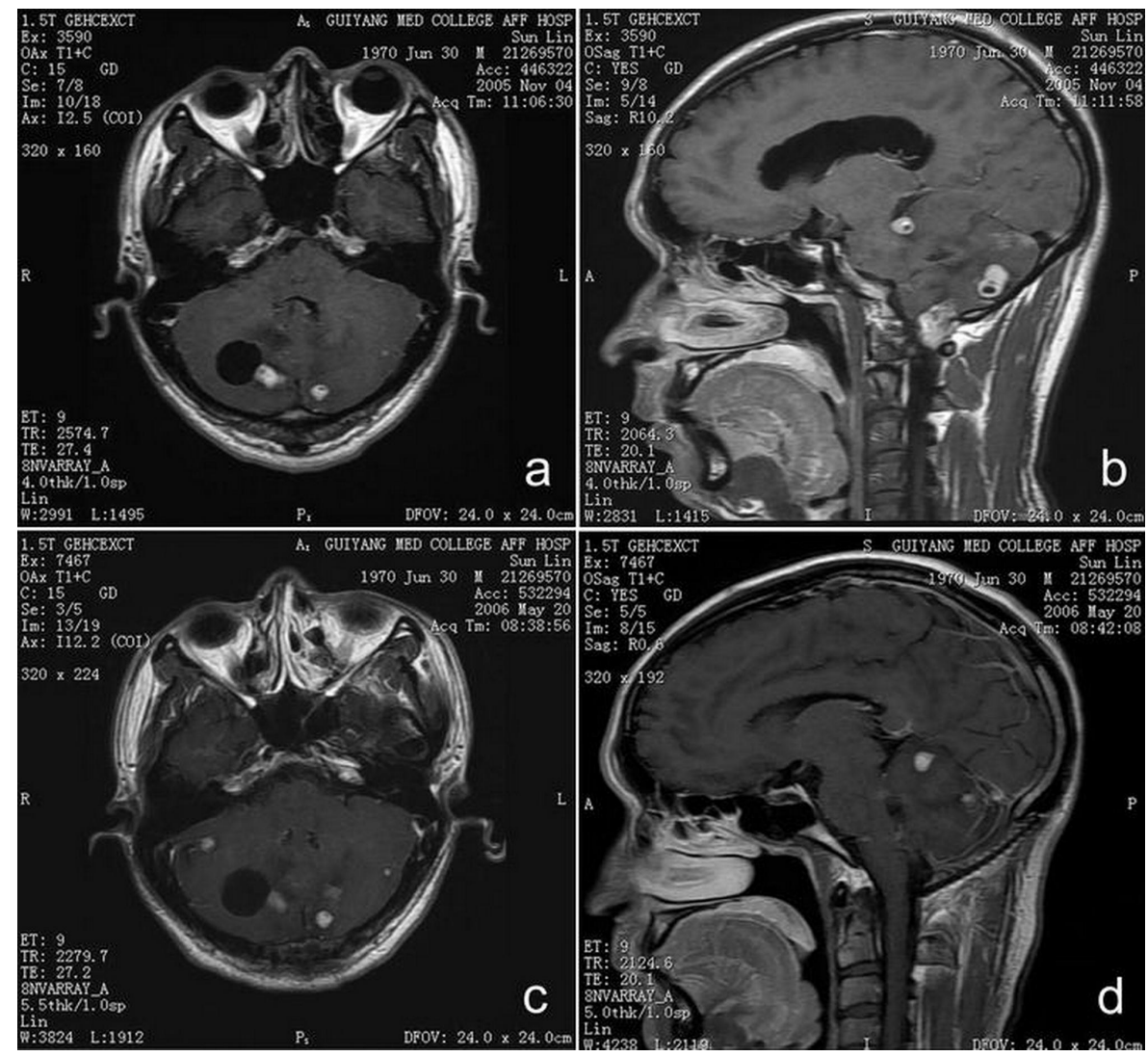

Figure 1. Performance of cranial magnetic resonance imaging before and after the first operation. a. The bilateral cerebellar hemispheres presented no abnormally enhanced foci. The right side exhibited cystic space occupation, and enhanced foci were apparent on the cyst wall. b. Sagittal plane: visibly enhanced foci, which differed in size in the cerebellar hemisphere and dorsal medulla. c. Reinspection post-operation: multiple abnormally enhanced foci recurred in the bilateral cerebellar hemispheres. d. Sagittal plane: morbidities in the dorsal medulla disappeared; nascent aggravated foci appeared in the cerebellar hemisphere. 


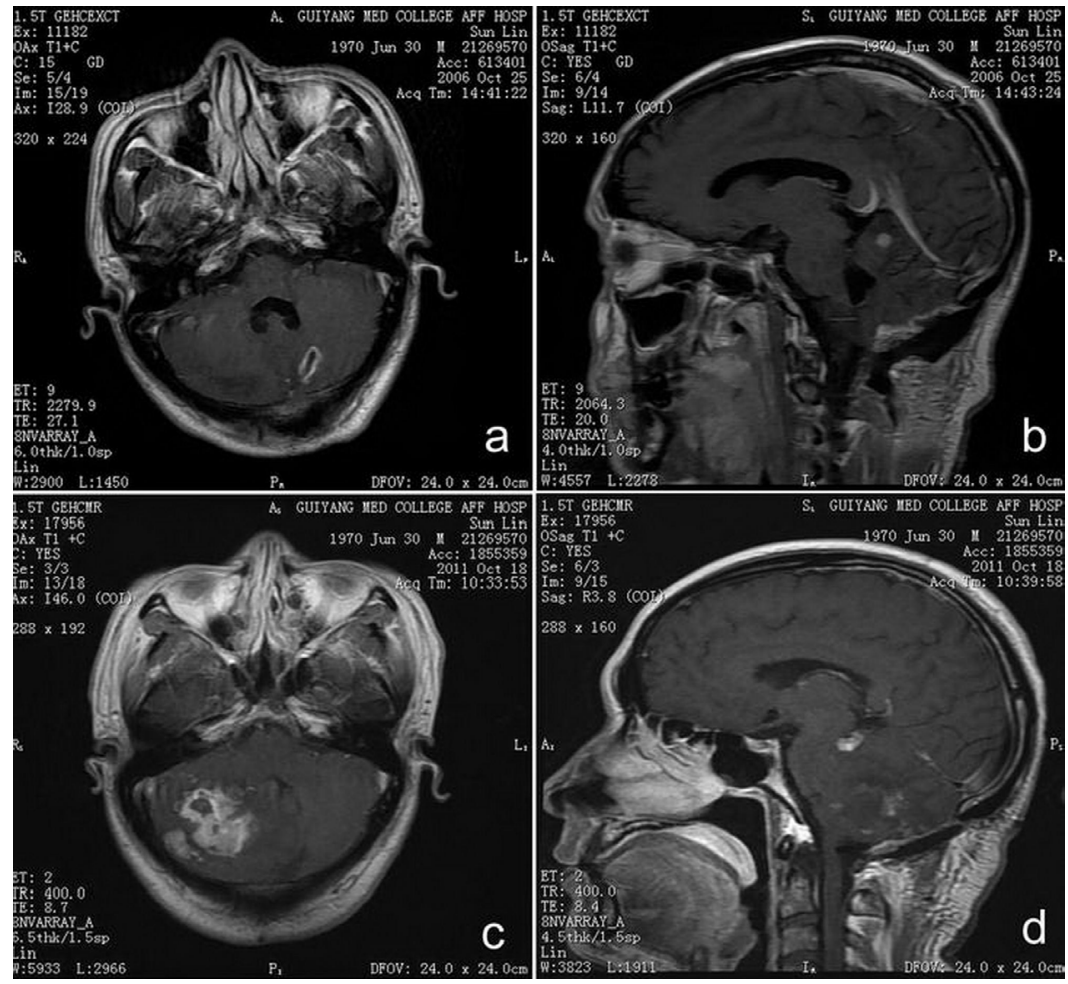

Figure 2. Performance of cranial magnetic resonance imaging before and after the second operation. a. Reinspection of the bilateral cerebellar hemispheres after the second operation revealed the disappearance of multiple abnormally enhanced foci. b. Sagittal plane: morbidities in the dorsal medulla disappeared; no obvious enhancement of a focus was observed in the cerebellar hemisphere. c. Reinspection of the right cerebellar hemisphere after the Gamma knife operation: tumors appeared to be lobulated. d. Reinspection after the Gamma knife operation: tumors regenerated in the cerebellar hemisphere and annular pool.
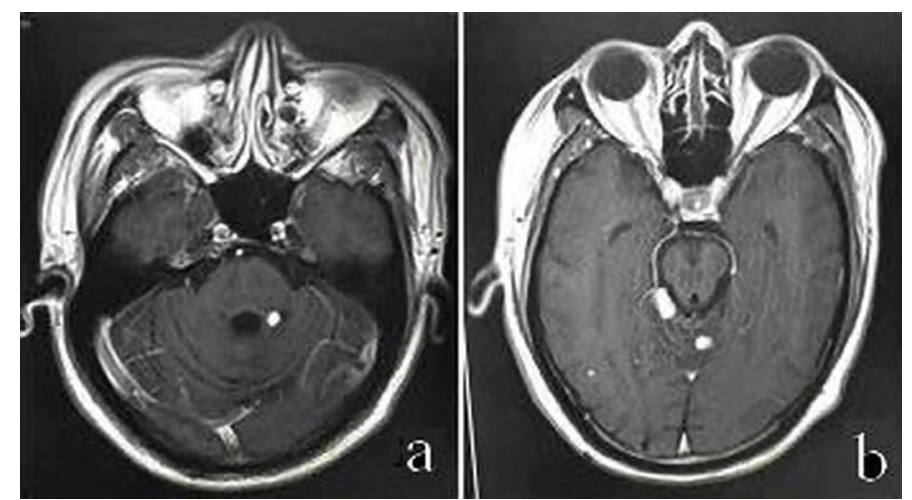

Figure 3. Performance of cranial magnetic resonance imaging after the third operation. a. Reinspection after the third operation: the focus in the cerebellar hemisphere disappeared, and no enhancement of foci was noted in the left annular pool. b. Reinspection of the cerebellar vermis after the third operation: no significant change in lesions was recorded in the annular pool. 


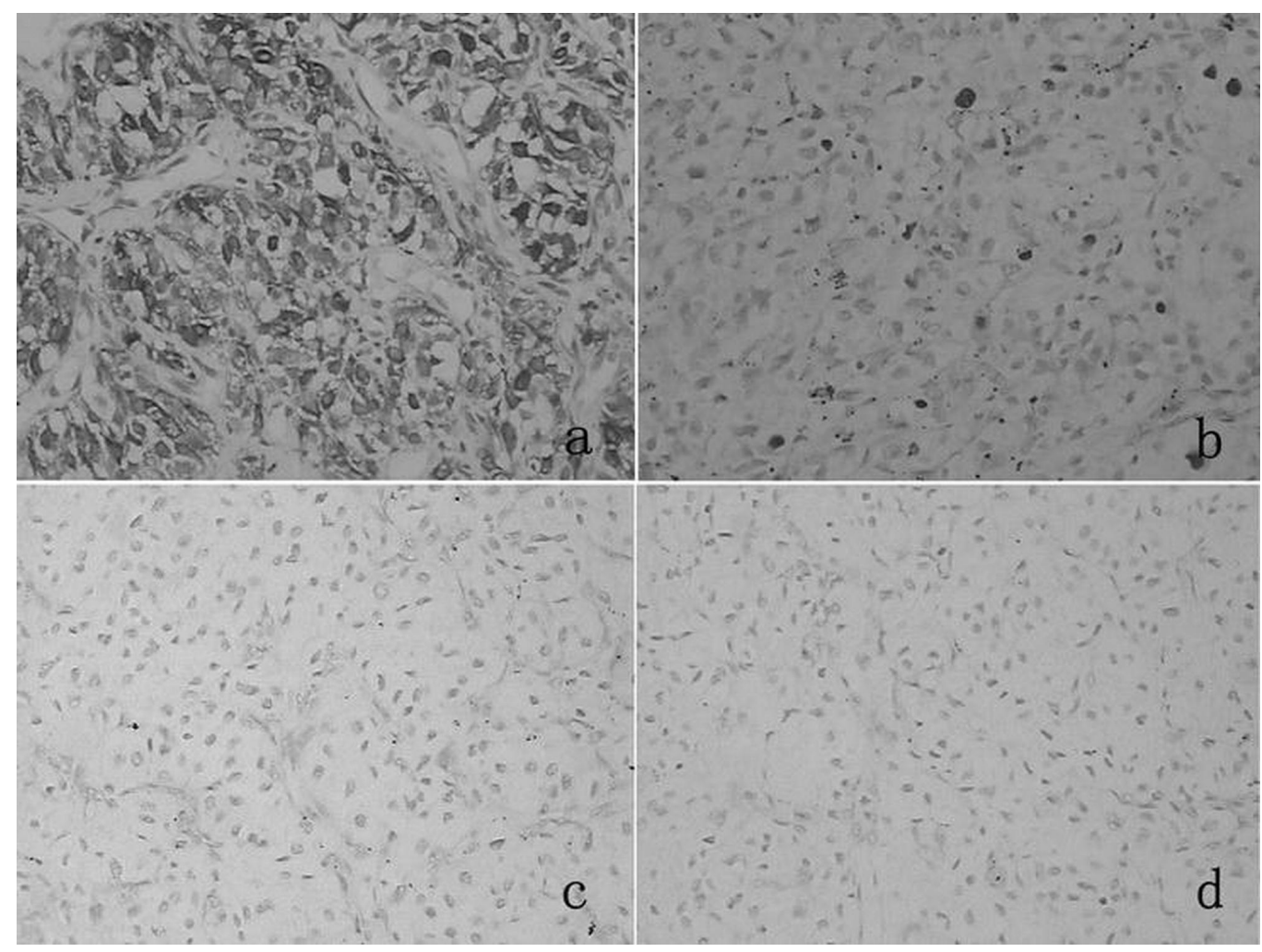

Figure 4. Immunohistochemical staining of the lesion tissue and control tissue (traumatic brain injury). a. Positive staining for $\alpha$-inhibin in lesion tissue (200X). b. Positive staining for Ki-67 in lesion tissue (200X). c. Negative staining for $\alpha$-inhibin in the control tissue (200X). d. Negative staining for Ki-67 in the control tissue (200X).

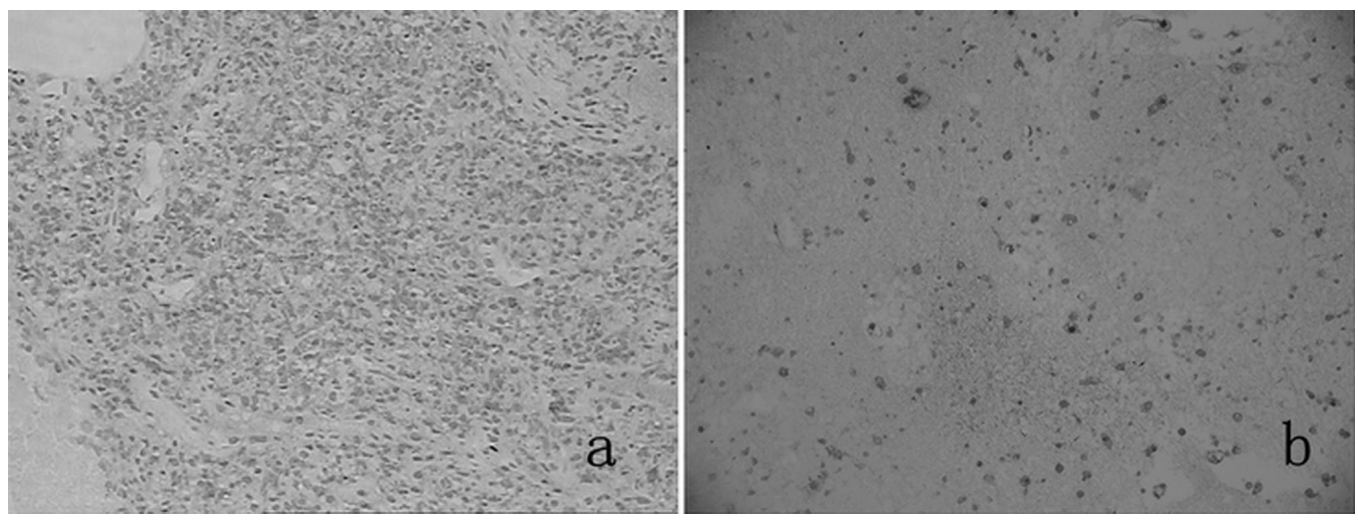

Figure 5. Vascular endothelial growth factor (VEGF) staining in the lesion tissue and control tissue. a. Positive staining for VEGF in lesion tissue (200X). b. Negative staining for VEGF in the control tissue (200X). 


\section{DISCUSSION}

Treatment plans for such cases may vary. First, some scholars believe that preoperative embolization of the feeding arteries can reduce blood loss and lower the risk of surgery (Tampieri et al., 1993; Vazquez-Anon et al., 1997). The blood supply to cerebral hemangioblastomas, however, is delivered primarily from the inferior arteries of the posterior and anterior cerebellum and the superior cerebellar artery branches. Because these vessels are always leptomeningeal vessels, embolization of these vessels during surgery is very dangerous. DSA testing indicated that the feeding artery of this patient was the inferior artery of the posterior cerebellum. Therefore, pre-operative embolization was not possible. Furthermore, although the efficacy of stereotactic radiosurgery against small and mediumsized solid cerebral hemangioblastomas is controversial (Rajaraman et al., 2004; Matsunaga et al., 2007), we extensively used this approach, and it was effective in practice. In addition, although the cyst wall is generally recognized as non-tumorous tissue that does not need to be excised, we agree that excision of whole cyst nodules including the cyst walls would help lower the incidence of recurrence (Conway et al., 2001). Therefore, we excised the cyst wall in this patient, although he had a relapse of the solid tumor at the nidus. Regardless of whether the cyst wall is strengthened, we believe that the cyst wall should be removed along with the nodule to prevent the growth of recurrent tumors.

The symptoms of the patient were prominently improved shortly after each operation. Although approximately $25 \%$ of patients with cerebral hemangioblastoma exhibit polycythemia (Richard et al., 1998), this finding was not observed in this patient. The solitary cystic nodule did not recur after excision. Three of the six solid neoplasms were not successfully extirpated; these lesions were small but tightly attached to the brain stem. During the operation, efforts to excise these lesions were unsuccessful. These three tumors grew significantly larger after the patient left the hospital. The tumor had several relapses in the cerebellar hemisphere 10-73 months after surgery. At present, the patient is in a good condition.

The VHL gene product, pVHL, has multiple functions. pVHL targets the alpha subunit of hypoxia inducible factor 1 (HIF1) to mediate ubiquitin-mediated proteolysis. HIF1 is involved in coordination of the cellular response to hypoxia and the transcriptional regulation of hypoxia-inducible genes, including VEGF, platelet-derived growth factor, transforming growth factor- $\alpha$, and erythropoetin. pVHL inactivation leads to HIF stabilization and subsequent transcriptional activation of HIF-induced target genes (Sivakumar et al., 2008). In patients with CNS HB, the increased expression of VEGF in tissues as determined by immunohistochemistry may be related to VHL gene deficiency. $\alpha$-inhibin was focally positive on immunohistochemistry and upregulated in stromal cells. The expression of Ki67 (marker of cellular proliferation) was decreased in the tumor cells. The upregulation of Ki67 and VEGF further proved the accuracy of this strategy for diagnosing cerebral hemangioblastoma. We believe that the high expression of these two factors predicts tumor recurrence after surgery.

In conclusion, the symptoms of the patient with multifocal CNS HB were prominently improved after each operation, suggesting that multifocal CNS HB should be treated by multiple surgeries followed by radiation therapy to inhibit the proliferation and relapse of these tumors. In addition, long-term regular hospital visits were useful. Furthermore, genetic diagnosis and gene-targeted therapy might be a promising strategy against familial CNS HB in the future. 


\title{
ACKNOWLEDGMENTS
}

\author{
Research supported by the Foundation of the Guizhou Province Science and Technology \\ Department [\#LG (2012) 053], and the Technology Bureau of Guiyang [\#(201203) 39].
}

\section{REFERENCES}

Conway JE, Chou D, Clatterbuck RE, Brem H, et al. (2001). Hemangioblastomas of the central nervous system in von Hippel-Lindau syndrome and sporadic disease. Neurosurgery 48: 55-62.

Jagannathan J, Lonser RR, Smith R, DeVroom HL, et al. (2008). Surgical management of cerebellar hemangioblastomas in patients with von Hippel-Lindau disease. J. Neurosurg. 108: 210-222.

Jarrell ST, Vortmeyer AO, Linehan WM, Oldfield EH, et al. (2006). Metastases to hemangioblastomas in von HippelLindau disease. J. Neurosurg. 105: 256-263.

Lee DK, Choe WJ, Chung CK and Kim HJ (2003). Spinal cord hemangioblastoma: surgical strategy and clinical outcome. J. Neurooncol. 61: 27-34.

Ma Z, Xie J and Zhao Y (1998). Hemangioblastomas of the central nervous system with von Hippel-Lindau's disease. Zhonghua Yi Xue Za Zhi 78: 460-461.

Matsunaga S, Shuto T, Inomori S, Fujino H, et al. (2007). Gamma knife radiosurgery for intracranial haemangioblastomas. Acta Neurochir. 149: 1007-1013.

Miyagami M and Katayama Y (2004). Long-term prognosis of hemangioblastomas of the central nervous system: clinical and immunohistochemical study in relation to recurrence. Brain Tumor Pathol. 21: 75-82.

Rajaraman C, Rowe JG, Walton L, Malik I, et al. (2004). Treatment options for von Hippel-Lindau's haemangioblastomatosis: the role of gamma knife stereotactic radiosurgery. Br. J. Neurosurg. 18: 338-342.

Rasmussen A, Nava-Salazar S, Yescas P, Alonso E, et al. (2006). Von Hippel-Lindau disease germline mutations in Mexican patients with cerebellar hemangioblastoma. J. Neurosurg. 104: 389-394.

Richard S, Campello C, Taillandier L, Parker F, et al. (1998). Haemangioblastoma of the central nervous system in von Hippel-Lindau disease. French VHL Study Group. J. Intern. Med. 243: 547-553.

Sivakumar V, Lu J, Ling EA and Kaur C (2008). Vascular endothelial growth factor and nitric oxide production in response to hypoxia in the choroid plexus in neonatal brain. Brain Pathol. 18: 71-85.

Tampieri D, Leblanc R and TerBrugge K (1993). Preoperative embolization of brain and spinal hemangioblastomas. Neurosurgery 33: 502-505.

Vazquez-Anon V, Botella C, Beltran A, Solera M, et al. (1997). Preoperative embolization of solid cervicomedullary junction hemangioblastomas: report of two cases. Neuroradiology 39: 86-89.

Woodward ER, Wall K, Forsyth J, MacDonald F, et al. (2007). VHL mutation analysis in patients with isolated central nervous system haemangioblastoma. Brain 130: 836-842. 